\title{
Long-Period Love Waves in a Heterogeneous, Spherical Earth ${ }^{1}$
}

\author{
Robert L. Kovach and Don L. Anderson \\ Jet Propulsion Laboratory and Seismological Laboratory \\ California Institute of Technology, Pasadena
}

\begin{abstract}
Periods of torsional eigenvibrations have been computed for heterogeneous spheres corresponding to a variety of earth models, and the periods of oscillation are used to calculate phase and group velocities for the fundamental and first higher modes of Love waves. A comparison is made between velocities computed for different spherical models and for equivalent flat eath structures. The comparison shows (1) that the effect of sphericity is more complicated for fundamental mode Love waves than for Rayleigh waves because of the efficient channeling of waves by low-velocity layers and (2) that the first higher Love mode is more affected by curvature than the fundamental mode. The variation with depth of the relative amplitude of the displacements indicates that the first higher Love mode for periods less than 90 seconds is very sensitive to upper-mantle structure in the vicinity of the low-velocity zone. Comparison of the theoretical results with recent phase velocity and torsional oscillation data shows that a Gutenberg type of velocity structure is more satisfactry than either the Lehmann or Jeffreys structures. The use of consistent densities with the Gutenberg model, rather than Bullen A densities, has a small but significant effect on the calculated velocities. For periods greater than 200 seconds the calculated phase velocities for various oceanic and continental structures are all within 2 per cent of each other. The calculated group velocities are within $1 / 2$ per cent of each other in the range $150<T<400 \mathrm{sec}$, thereby confirming experimental results. Dispersion measurements must therefore be made with precision if significant conclusions are to be inferred about details of earth structure.
\end{abstract}

Introduction. There have been many recent studies, both observational and theoretical, on the dispersion of long-period Rayleigh waves in the earth. In an important paper, Dorman et al. [1960] presented extensive computations to explain observed mantle Rayleigh wave dispersion. They calculated Rayleigh wave dispersion for eleven models of continental and oceanic structure for a flat, layered earth using the Thomson-Haskell matrix formulation. From data of Exing and Press $[1954 a, b]$ they concluded that the mantle structure under continents proposed by Gutenberg [see Bullard, 1957] was far superior to the standard Jeffreys-Bullen structure. It was also shown that a modification of a mantle structure proposed by Lehmann [1955] was consistent with Pacinc Ocean data. Both the Gutenberg and the Lehmann models include a low-velocity zone in the upper mantle. Takeuchi, Press, and Kobayashi [1959] used a variational method and showed that Rayleigh wave dispersion data required the existence of

\footnotetext{
${ }^{1}$ Contribution 1101, Division of the Geological Sicnces, California Institute of Technology.
}

Gutenberg's low-velocity zone in the upper mantle. Aki and Press [1961], using a synthetic seismogram approach, demonstrated that the Atlantic and Indian oceans also had low-velocity zones and presented an alternative model for the $\mathrm{Pa}$ cific Ocean.

Calculations based on flat earth models and Rayleigh wave group velocity data for periods between 50 and 250 seconds were used in all these fundamental studies. An important question was the influence of gravity and sphericity in this range of periods. This question was answered by Bolt and Dorman [1961] and by Alterman et al. [1961]. By numerical integration of the equations of spheroidal motion for four models of a spherical, gravitating earth, Bolt and Dorman concluded that the combined effect of gravity and sphericity on phase velocity could not be ignored for Rayleigh waves with periods greater than about 50 seconds, but that group velocities for $100<T<250$ seconds were accurate to 1 per cent. The general conclusions of the earlier papers, being based on group velocity data, therefore remained correct. Bolt and Dorman further demonstrated 


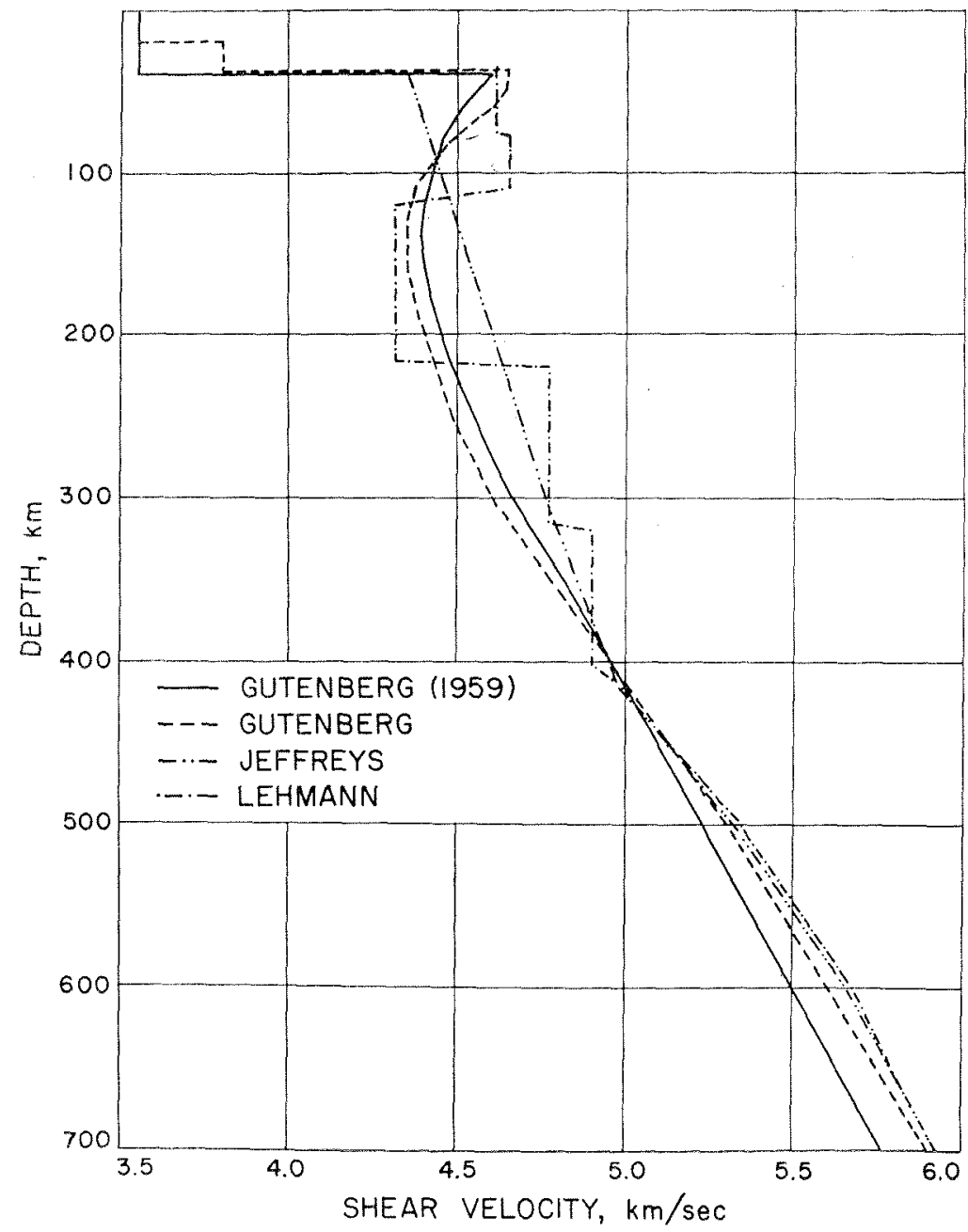

Fig. 1, Shear wave velocity distributions for continental models.

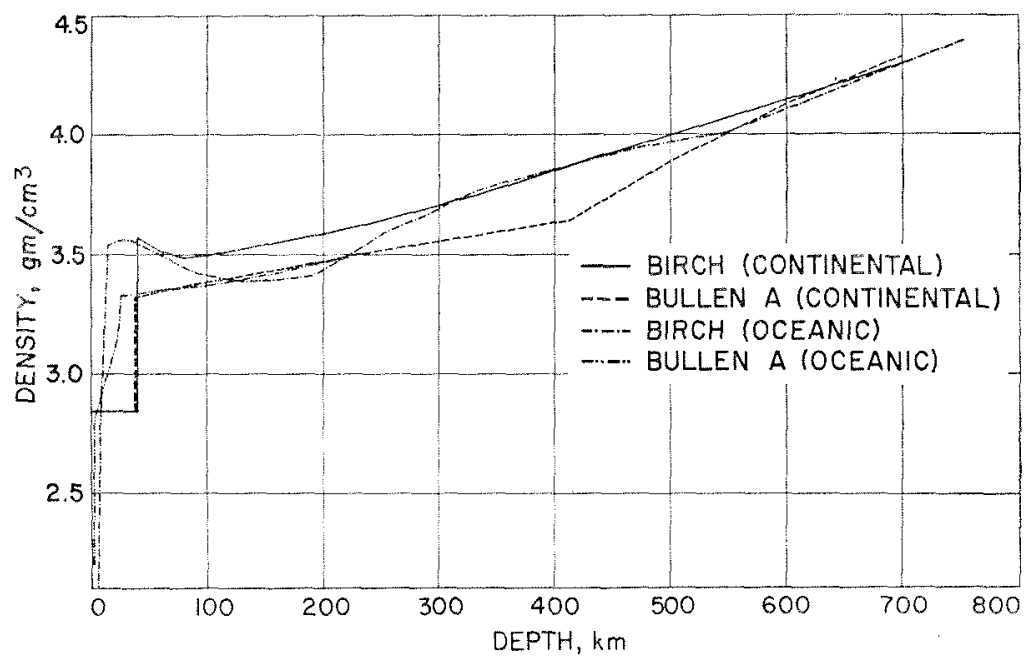

Fig. 2. Density distributions for continental and oceanic models. 
TABLE 1. Parameters for Gutenberg-Birch Model

\begin{tabular}{|c|c|c|c|}
\hline$R / R_{0}{ }^{*}$ & $\beta, \mathrm{km} / \mathrm{sec}$ & $\rho, \mathrm{g} / \mathrm{cm}^{3}$ & $\begin{array}{c}\mu, \text { dynes } \\
\mathrm{cm}^{-2} \times 10^{11}\end{array}$ \\
\hline 1.0000 & 3.55 & 2.84 & 3.579 \\
\hline .9940 & 3.55 & 2.84 & 3.579 \\
\hline 9940 & 4.60 & 3.57 & 7.554 \\
\hline .9906 & 4.51 & 3.507 & 7.133 \\
\hline 9874 & 4.45 & 3.486 & 6.903 \\
\hline .9843 & 4.42 & 3.495 & 6.828 \\
\hline 9812 & 4.40 & 3.513 & 6.801 \\
\hline .9780 & 4.39 & 3.528 & 6.799 \\
\hline 9749 & 4.40 & 3.546 & 6.865 \\
\hline 9717 & 4.42 & 3.564 & 6.963 \\
\hline 9686 & 4.45 & 3.582 & 7.093 \\
\hline .9655 & 4.48 & 3.606 & 7.237 \\
\hline 9623 & 4.52 & 3.628 & 7.412 \\
\hline .9592 & 4.565 & 3.652 & 7.610 \\
\hline 9561 & 4.61 & 3.676 & 7.812 \\
\hline .9529 & 4.66 & 3.700 & 8.035 \\
\hline 9451 & 4.81 & 3.773 & 8.729 \\
\hline .9372 & 4.95 & 3.848 & 9.429 \\
\hline 9294 & 5.09 & 3.924 & 10.166 \\
\hline .9215 & 5.22 & 3.996 & 10.888 \\
\hline .9137 & 5.36 & 4.071 & 11.696 \\
\hline .9058 & 5.50 & 4.147 & 12.545 \\
\hline .8901 & 5.77 & 4.301 & 14.319 \\
\hline .8744 & 6.04 & 4.422 & 16.132 \\
\hline .8587 & 6.30 & 4.543 & 18.031 \\
\hline .8430 & 6.35 & 4.573 & 18.439 \\
\hline .8116 & 6.50 & 4.694 & 19.832 \\
\hline .7803 & 6.60 & 4.769 & 20.774 \\
\hline .7489 & 6.75 & 4.845 & 22.075 \\
\hline .7175 & 6.85 & 4.920 & 23.086 \\
\hline .6861 & 6.95 & 4.996 & 24.132 \\
\hline .6547 & 7.00 & 5.056 & 24.774 \\
\hline .6233 & 7.10 & 5.116 & 25.790 \\
\hline .5919 & 7.20 & 5.192 & 26.915 \\
\hline .5605 & 7.25 & 5.267 & 27.685 \\
\hline .5448 & 7.20 & 5.267 & 27.304 \\
\hline .5417 & 7.20 & 5.252 & 27.226 \\
\hline
\end{tabular}

${ }^{*} R_{0}=6371 \mathrm{~km}$ in Tables $1-3$.

that a Gutenberg velocity structure with Bullen A densities is consistent with phase and group velocity data to 300 seconds period.

Alterman et al. [1961] also showed that calculations for a flat earth gave phase velocities correct to 1 per cent only to 50 seconds period and that group velocities were correct to 1 per cent to 250 seconds. Their solutions for a spherical earth also favored the Gutenberg mantle structure.

An equivalent study of mantle Love waves has not yet been presented. Published resultsdata and theory-are inconclusive. In a prelimi-

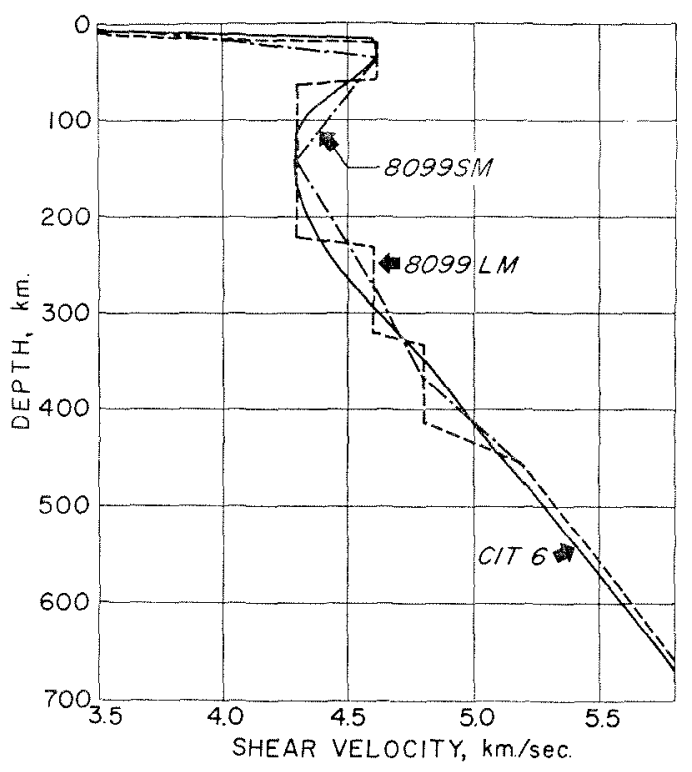

Fig. 3. Shear wave velocity distributions for oceanic models.

TABLE 2. Parameters for 8099 Models

$\begin{array}{ccccc}R / R_{0} & R / R_{0} & \beta, & \rho, & \mu, \text { dynes } \\ 8099 \mathrm{LM} & 8099 \mathrm{SM} & \mathrm{km} / \mathrm{sec} & \mathrm{g} / \mathrm{cm}^{3} & \mathrm{~cm}^{-2} \times 10^{11}\end{array}$

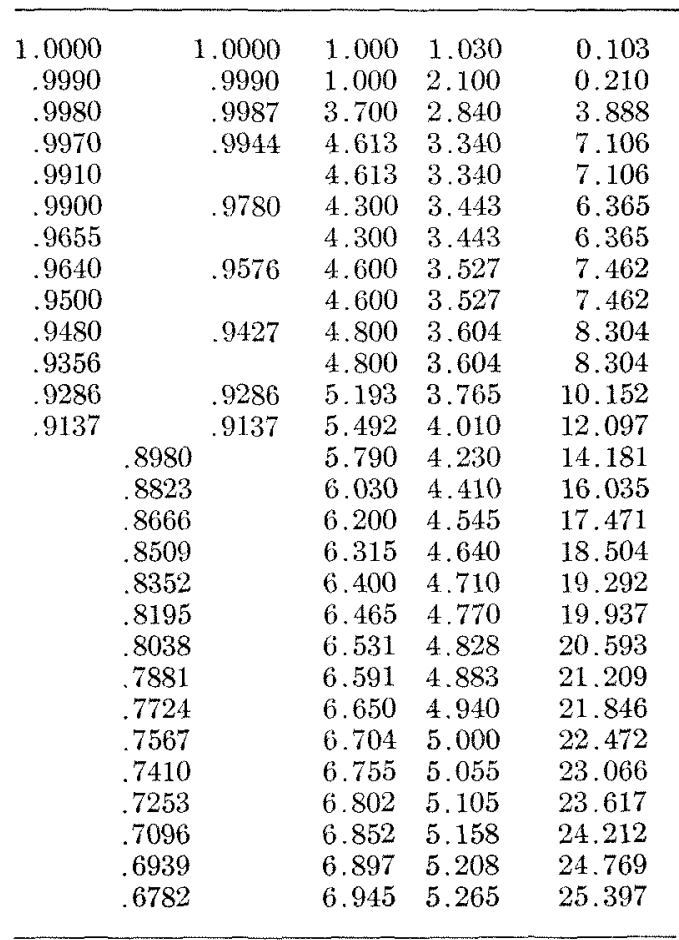


TABLE 3. Parameters for CIT6 Oceanic Model

\begin{tabular}{|c|c|c|c|}
\hline$R / R_{0}$ & $\beta, \mathrm{km} / \mathrm{sec}$ & $\rho, \mathrm{g} / \mathrm{cm}^{3}$ & $\begin{array}{c}\mu, \text { dynes } \\
\mathrm{cm}^{-2} \times 10^{11}\end{array}$ \\
\hline 1.0000 & 1.000 & 1.000 & 0.100 \\
\hline .9996 & 1.000 & 1.000 & 0.100 \\
\hline .9991 & 1.000 & 2.100 & 0.210 \\
\hline .9987 & 3.700 & 2.840 & 3.888 \\
\hline .9976 & 4.600 & 3.535 & 7.480 \\
\hline .9965 & 4.612 & 3.555 & 7.560 \\
\hline .9957 & 4.612 & 3.555 & 7.560 \\
\hline .9945 & 4.609 & 3.550 & 7.540 \\
\hline .9922 & 4.560 & 3.520 & 7.320 \\
\hline .9890 & 4.450 & 3.470 & 6.870 \\
\hline .9859 & 4.339 & 3.420 & 6.440 \\
\hline .9827 & 4.300 & 3.400 & 6.287 \\
\hline .9796 & 4.290 & 3.390 & 6.240 \\
\hline .9765 & 4.290 & 3.390 & 6.240 \\
\hline .9733 & 4.301 & 3.400 & 6.290 \\
\hline .9702 & 4.322 & 3.410 & 6.370 \\
\hline .9670 & 4. 360 & 3.462 & 6.581 \\
\hline .9639 & 4.402 & 3.515 & 6.810 \\
\hline .9608 & 4.460 & 3.585 & 7.130 \\
\hline .9576 & 4.521 & 3.625 & 7.410 \\
\hline .9513 & 4.661 & 3.720 & 8.080 \\
\hline .9482 & 4.741 & 3.760 & 8.450 \\
\hline .9451 & 4.824 & 3.790 & 8.820 \\
\hline .9403 & 4.911 & 3.830 & 9.238 \\
\hline .9333 & 5.040 & 3.890 & 9.880 \\
\hline .9254 & 5.210 & 3.950 & 10.722 \\
\hline .9137 & 5.450 & 4.010 & 11.910 \\
\hline .8980 & 5.761 & 4.210 & 13.970 \\
\hline .8823 & 6.030 & 4.400 & 16.000 \\
\hline .8666 & 6.230 & 4.560 & 17.700 \\
\hline .8509 & 6.322 & 4.630 & 18.504 \\
\hline .8273 & 6.421 & 4.740 & 19.540 \\
\hline .7959 & 6.550 & 4.850 & 20.810 \\
\hline .7645 & 6.690 & 4.960 & 22.200 \\
\hline .7332 & 6.780 & 5.070 & 23.306 \\
\hline .7018 & 6.900 & 5.190 & 24.710 \\
\hline .6704 & 6.97 & 5.290 & 25.70 \\
\hline .6390 & 7.05 & 5.390 & 26.79 \\
\hline .6076 & 7.15 & 5.490 & 28.07 \\
\hline .5762 & 7.23 & 5.590 & 29.22 \\
\hline .5528 & 7.20 & 5.690 & 29.50 \\
\hline
\end{tabular}

nary note, Satô et al. [1960] presented theoretical results for Love waves in a spherical earth with a Jeffreys-Bullen A structure. No comparison was made with data. Takeuchi [1959], Gitbert and MacDonald [1960], MacDonald and Ness [1961], and Pekeris et al. [1961] also computed theoretical torsional oscillation periods. MacDonald and Ness concluded that a modified Gutenberg mantle fits the torsional oscillation data best, although the range of periods considered was not sensitive to details of the upper mantle structure. Kobayashi and Takeuchi
[1961], using calculations for a flat earth, concluded that the Jeffrey's model gave better agreement than the Gutenberg model for mantle Love waves. Jobert [1960] also computed dispersion of Love waves on a spherical earth for several structures of continental and oceanic type.

Because a knowledge of Love wave dispersion gives valuable information about the shear wave velocity variations in the earth, extensive calculations are presented here in an examination of the sensitivity of Love waves to variations in assumed earth models. The sensitivity of Love waves to variations in internal structure is an important question not only for terrestrial seismology but also for future planetary exploration.

The method used to obtain the new Love wave velocities depends on the calculation of the periods of the free torsional modes of vibration for a heterogeneous, elastic sphere. An outline of the method was presented by Alter-

TABLE 4. Jeffreys-Bullen A Model

\begin{tabular}{|c|c|c|}
\hline Order, $n$ & $T$, sec & $c, \mathrm{~km} / \mathrm{sec}$ \\
\hline 18 & 386.67 & 5.595 \\
\hline 20 & 355.60 & 5.490 \\
\hline 22 & 329.42 & 5.400 \\
\hline 24 & 307.01 & 5.321 \\
\hline 25 & 296.96 & 5.286 \\
\hline 26 & 287.57 & 5.252 \\
\hline 28 & 270.53 & 5.191 \\
\hline 30 & 255.46 & 5.137 \\
\hline 32 & 242.02 & 5.088 \\
\hline 34 & 229.96 & 5.045 \\
\hline 36 & 219.07 & 5.005 \\
\hline 38 & 209.19 & 4.970 \\
\hline 40 & 200.18 & 4.937 \\
\hline 42 & 191.93 & 4.907 \\
\hline 44 & 184.34 & 4.879 \\
\hline 46 & 177.34 & 4.853 \\
\hline 48 & 170.87 & 4.830 \\
\hline 50 & 164.86 & 4.807 \\
\hline 52 & 159.27 & 4.787 \\
\hline 54 & 154.05 & 4.767 \\
\hline 56 & 149.17 & 4.749 \\
\hline 58 & 144.60 & 4,731 \\
\hline 60 & 140.31 & 4.715 \\
\hline 62 & 136.27 & 4.699 \\
\hline 64 & 132.46 & 4.684 \\
\hline 66 & 128.87 & 4.670 \\
\hline 68 & 125.47 & 4.657 \\
\hline 70 & 122.25 & 4.644 \\
\hline 72 & 119.23 & 4.630 \\
\hline 74 & 116.33 & 4.618 \\
\hline 76 & 113.58 & 4.607 \\
\hline
\end{tabular}


TABLE 5. Lehmann Model

\begin{tabular}{|c|c|c|}
\hline Order, $n$ & $T$, sec & $c, \mathrm{~km} / \mathrm{sec}$ \\
\hline 18 & 386.81 & 5.593 \\
\hline 20 & 355.67 & 5.489 \\
\hline 22 & 329.44 & 5.400 \\
\hline 24 & 306.97 & 5.322 \\
\hline 25 & 296.89 & 5.287 \\
\hline 26 & 287.48 & 5.254 \\
\hline 28 & 270.39 & 5.194 \\
\hline 30 & 255.27 & 5.141 \\
\hline 32 & 241.78 & 5.093 \\
\hline 34 & 229.68 & 5.051 \\
\hline 36 & 218.74 & 5.013 \\
\hline 38 & 208.82 & 4.978 \\
\hline 40 & 199.77 & 4.947 \\
\hline 42 & 191.47 & 4.918 \\
\hline 44 & 183.85 & 4.892 \\
\hline 46 & 176.82 & 4.868 \\
\hline 48 & 170.31 & 4.846 \\
\hline 50 & 164.26 & 4.825 \\
\hline 52 & 158.64 & 4.806 \\
\hline 54 & 153.39 & 4.788 \\
\hline 58 & 143.87 & 4.755 \\
\hline 60 & 139.55 & 4.741 \\
\hline 62 & 135.48 & 4.727 \\
\hline 64 & 131.65 & 4.714 \\
\hline 66 & 128.02 & 4.701 \\
\hline 68 & 124.60 & 4.689 \\
\hline 70 & 121.35 & 4.678 \\
\hline 72 & 118.27 & 4.668 \\
\hline 74 & 115.35 & 4.657 \\
\hline 76 & 112.57 & 4.648 \\
\hline 78 & 109.92 & 4.639 \\
\hline 80 & 107.40 & 4.630 \\
\hline 84 & 102.58 & 4.618 \\
\hline
\end{tabular}

man et al. [1959]; it is based on earlier analyses by Love [1911], Hoskins [1920], and Jeans [1923]. Stoneley [1961] presented an excellent review of the earlier calculations. Other techniques used to isolate the torsional eigenvibrations have been the variational method [Jobert, 1956; Takeuchi, 1959], an extension of the Thomson-Haskell matrix method [Gilbert and MacDonald, 1960], and a direct numerical integration of the equations of motion [Satô et al., 1960]. Only a limited number of models have been considered in the previous papers, and the main attention has been focused on the low-order oscillations. There is significant disagreement between many of the published values of vibration periods.

Since any eigenvalue problem requires a large amount of computation time, the results of the calculations are tabulated here in detail. The results can be used for studying not only the dispersion of Love waves but the free torsional oscillations themselves.

Computations are presented for the fundamental Love mode of periods between 60 and about 600 seconds and are compared with recent phase velocity data. Comparisons of calculations for flat and spherical surfaces with equivalent structures are made, and the first higher Love mode is investigated for continental and oceanic structures.

Numerical calculations and verification of results. Alterman et al. [1959] have shown that the torsional oscillations can be defined by the system of equations

$$
\frac{d y_{1}}{d x}=\frac{1}{x} y_{1}+\frac{a}{\mu(x)} y_{2}
$$

$\frac{d y_{2}}{d x}$

$$
=\left[\frac{\mu(x)\left(n^{2}+n-2\right)}{a x^{2}}-a \sigma^{2} \rho_{0}(x)\right] y_{1}-\frac{3}{x} y_{2}
$$

where

$$
\begin{aligned}
a & =\text { radius of spherical body. } \\
x & =\text { normalized radius. } \\
\mu(x) & =\text { rigidity. } \\
\rho_{0}(x) & =\text { unperturbed density. } \\
n & =\text { order number of spherical harmonic. } \\
\sigma & =\text { frequency. } \\
y_{1} & =\text { radial factor of the displacements. } \\
y_{2} & =\text { radial factor of the shear stresses. }
\end{aligned}
$$

This system of equations was solved by Carr [1961] for a solid sphere and was coded in Fortran for an IBM 7090 computer. Sinee we are restricting our discussion to oscillations that are confined to the mantle, the presence of a liquid core is of no concern in the immediate problem. However, the boundary conditions are slightly changed for a solid sphere because regularity at the origin must be satisfied, in addition to the vanishing of stresses at the free surface.

Because the method of solution is thoroughly discussed by Carr [1961], we shall only briefly outline the numerical solution here. The AdamsMoulton predictor-corrector method is used in integrating the differential equations downward from the free surface. Runge-Kutta-Gill formulas are used to start the integration process and are used to restart the integration whenever the step size has been changed. The integration 
TABLE 6. Gutenberg Model

\begin{tabular}{ccc}
\hline Order, $n$ & $T, \mathrm{sec}$ & $c, \mathrm{~km} / \mathrm{sec}$ \\
\hline 18 & 391.18 & 5.531 \\
20 & 359.91 & 5.426 \\
22 & 333.52 & 5.334 \\
24 & 310.89 & 5.256 \\
25 & 300.74 & 5.220 \\
26 & 291.24 & 5.187 \\
28 & 274.00 & 5.126 \\
30 & 258.73 & 5.073 \\
32 & 245.09 & 5.025 \\
34 & 232.85 & 4.983 \\
36 & 221.78 & 4.945 \\
38 & 211.73 & 4.910 \\
40 & 202.56 & 4.880 \\
42 & 194.15 & 4.851 \\
44 & 186.42 & 4.826 \\
46 & 179.28 & 4.802 \\
48 & 172.67 & 4.780 \\
50 & 166.53 & 4.760 \\
52 & 160.82 & 4.741 \\
54 & 155.49 & 4.724 \\
56 & 150.50 & 4.708 \\
58 & 145.82 & 4.693 \\
60 & 141.43 & 4.679 \\
62 & 137.29 & 4.665 \\
64 & 133.39 & 4.653 \\
66 & 129.70 & 4.641 \\
68 & 126.22 & 4.630 \\
70 & 122.92 & 4.619 \\
72 & 119.79 & 4.609 \\
74 & 116.81 & 4.600 \\
76 & 113.98 & 4.591 \\
78 & 111.29 & 4.582 \\
80 & 108.72 & 4.574 \\
\hline & &
\end{tabular}

step size is variable and is controlled internally by specifying that the truncation error shall not exceed a prescribed bound. Partial double precision is used to control the growth of round-off error. Input data are given in a table of normalized radius, rigidity, and density. Intermediate values needed for computation are obtained internally by linear interpolation.

Regularity at the origin was met by a power series expansion for the two dependent variables $y_{1}$ and $y_{2}$; within the radius of convergence of the power series it is required that the solutions of the differential equations and of the power series match. This requirement gives rise to a characteristic determinant which equals zero for the correct eigenfrequency $\sigma$. A sequence of approximations for $\sigma$ is used, halving the sum of the previous calculations, which makes the characteristic determinant change sign. The process is terminated when the value of $\sigma$ is un- changed up to a specified number of significant digits.

Verification of the numerical accuracy of the program was accomplished in several ways. The periods of oscillation for $n=2,3$, and 4 for a homogeneous moon model were ealculated [Carr and Kovach, 1962] and were found to agree exactly with the published values of Takeuchi, Saito, and Kobayashi [1961] obtained by independent means. Our calculations agree to three significant digits with published values of Sato et al. [1960] for the Jeffreys-Bullen model and with published values of Pekeris et al. [1961] for the Gutenberg model.

Earth models. For the computations presented here, the earth is assumed to consist of

TABLE 7. Gutenberg-Birch Model

\begin{tabular}{|c|c|c|}
\hline Order, $n$ & $T$, sec & $c, \mathrm{~km} / \mathrm{sec}$ \\
\hline 18 & 393.10 & 5.505 \\
\hline 20 & 361.29 & 5.405 \\
\hline 22 & 334.48 & 5.319 \\
\hline 24 & 311.54 & 5.245 \\
\hline 26 & 291.65 & 5.179 \\
\hline 28 & 274.23 & 5.122 \\
\hline 30 & 258.82 & 5.071 \\
\hline 32 & 245.09 & 5.025 \\
\hline 34 & 232.78 & 4.985 \\
\hline 36 & 221.66 & 4.948 \\
\hline 38 & 211.58 & 4.914 \\
\hline 40 & 202.39 & 4.884 \\
\hline 42 & 193.98 & 4.856 \\
\hline 44 & 186.24 & 4.830 \\
\hline 46 & 179.11 & 4.806 \\
\hline 48 & 172.51 & 4.784 \\
\hline 50 & 166.39 & 4.764 \\
\hline 52 & 160.70 & 4.745 \\
\hline 54 & 155.38 & 4.727 \\
\hline 56 & 150.41 & 4.710 \\
\hline 58 & 145.76 & 4.695 \\
\hline 60 & 141.38 & 4.680 \\
\hline 62 & 137.27 & 4.666 \\
\hline 64 & 133.39 & 4.653 \\
\hline 66 & 129.73 & 4.640 \\
\hline 68 & 126.27 & 4.628 \\
\hline 70 & 122.99 & 4.617 \\
\hline 72 & 119.88 & 4.606 \\
\hline 74 & 116.92 & 4.596 \\
\hline 76 & 114.11 & 4.586 \\
\hline 78 & 111.43 & 4.576 \\
\hline 80 & 108.88 & 4.567 \\
\hline 82 & 106.45 & 4.558 \\
\hline 84 & 104.13 & 4.550 \\
\hline 86 & 101.90 & 4.541 \\
\hline 88 & 99.77 & 4.534 \\
\hline 90 & 97.73 & 4.526 \\
\hline
\end{tabular}


TABLE 8. 8099LM Model

\begin{tabular}{ccc}
\hline Order, $n$ & T, see & $c, \mathrm{~km} / \mathrm{sec}$ \\
\hline 16 & 429.06 & 5.654 \\
18 & 391.30 & 5.529 \\
20 & 359.95 & 5.424 \\
22 & 333.59 & 5.332 \\
24 & 311.01 & 5.253 \\
25 & 300.85 & 5.217 \\
26 & 291.35 & 5.184 \\
28 & 274.09 & 5.124 \\
30 & 258.81 & 5.070 \\
32 & 245.17 & 5.023 \\
34 & 232.91 & 4.981 \\
36 & 221.83 & 4.943 \\
38 & 211.76 & 4.909 \\
40 & 202.57 & 4.879 \\
42 & 194.15 & 4.851 \\
44 & 186.40 & 4.825 \\
46 & 179.25 & 4.802 \\
48 & 172.63 & 4.780 \\
50 & 166.48 & 4.761 \\
52 & 160.75 & 4.742 \\
54 & 155.41 & 4.726 \\
56 & 150.41 & 4.710 \\
58 & 145.72 & 4.695 \\
\hline
\end{tabular}

spherical shells of variable thickness. Each shell has linear gradients of velocity and density. Therefore, any velocity-density distribution can be approximated as closely as is desired by increasing the number of entries in the input. tables. Seven models of the earth's mantle are considered; four models are continental and three are oceanic.

The continental models are the GutenbergBullen A, the Jeffreys-Bullen A, the LehmannBullen $A$, and the Gutenberg-Birch [Gutenberg, 1959]. The Gutenberg-Bullen A model is the same as that considered by Peleris et al. [1961]. Velocity-density parameters for the JeffreysBullen $A$ and the Lehmann-Bullen A models were taken from Satô et al. [1960]. Figures 1 and 2 show the shear velocity and density distributions for the continental models.

Because the Gutenberg-Bullen A and the Lehmann-Bullen A models contain inconsistent velocity-density combinations, an additional earth model designated the Gutenberg-Birch model was constructed. This model is based on the most recent results of compressional and shear velocity obtained by Gutenberg [1959] and has slightly higher shear velocities in the low-velocity zone (Figure 1) than the familiar Gutenberg model. Density was obtained from the compres- sional velocity-density relation $\rho=1.13+$ $0.302 V_{P}$ given by Birch [1961]. This relation is consistent with a mantle of mean atomic weight 22.5 , and it gives a density reversal in the lowvelocity zone (Figure 2 ). The variation of the physical parameters for the Gutenberg-Birch model is given in Table 1. Anderson and Harkrider [1962] have shown from calculations for a flat earth that the difference between Bullen A and Birch densities has only a slight effect on Rayleigh waves and an almost negligible effect on Love waves for periods less than 300 seconds.

Two of the oceanic models considered are versions of Dorman's model 8099 . The model 8099LM (Figure 3, Table 2) approximates the actual layering used in the calculations for a flat earth, whereas 8099SM (Table 2) is constructed with straight-line segments joining layer midpoints. CIT6 (Table 3) is a smoother structure with a low-velocity channel of the Gutenberg type and a density structure of the Birch type (Figures 2 and 3). These three similar models allow us to investigate the sensitivity of mantle Love waves to details in the upper mantle.

TABLE 9. 8099sM Model

\begin{tabular}{|c|c|c|}
\hline Order, $n$ & $T$, sec & $c, \mathrm{~km} / \mathrm{sec}$ \\
\hline 14 & 472.75 & 5.839 \\
\hline 16 & 425.83 & 5.696 \\
\hline 18 & 388.08 & 5.575 \\
\hline 20 & 356.85 & 5.471 \\
\hline 22 & 330.47 & 5.383 \\
\hline 25 & 297.85 & 5.270 \\
\hline 26 & 288.39 & 5.237 \\
\hline 27 & 279.54 & 5.207 \\
\hline 28 & 271.21 & 5.178 \\
\hline 29 & 263.39 & 5.151 \\
\hline 30 & 255.99 & 5.126 \\
\hline 31 & 249.00 & 5.103 \\
\hline 32 & 242.41 & 5.080 \\
\hline 33 & 236.18 & 5.059 \\
\hline 34 & 230.27 & 5.038 \\
\hline 35 & 224.64 & 5.019 \\
\hline 36 & 219.28 & 5.001 \\
\hline 37 & 214.17 & 4.984 \\
\hline 38 & 209.29 & 4.967 \\
\hline 39 & 204.63 & 4.952 \\
\hline 40 & 200.18 & 4.937 \\
\hline 50 & 164.41 & 4.821 \\
\hline 60 & 139.49 & 4.743 \\
\hline 75 & 113.65 & 4.664 \\
\hline 80 & 107.04 & 4.645 \\
\hline 90 & 95.89 & 4.612 \\
\hline
\end{tabular}


TABIE 10. CIT6 Model

\begin{tabular}{|c|c|c|}
\hline Order, $n$ & $T$, sec & $c, \mathrm{~km} / \mathrm{sec}$ \\
\hline 14 & 475.62 & 5.804 \\
\hline 16 & 428.42 & 5.662 \\
\hline 18 & 390.38 & 5.542 \\
\hline 20 & 358.93 & 5.440 \\
\hline 22 & 332.39 & 5.352 \\
\hline 24 & 309.69 & 5.275 \\
\hline 26 & 289.97 & 5.209 \\
\hline 28 & 272.68 & 5.150 \\
\hline 30 & 257.37 & 5.099 \\
\hline 32 & 243.72 & 5.053 \\
\hline 34 & 231.46 & 5.012 \\
\hline 35 & 225.78 & 4.993 \\
\hline 36 & 220.38 & 4.976 \\
\hline 38 & 210.33 & 4.943 \\
\hline 40 & 201.16 & 4.913 \\
\hline 42 & 192.75 & 4.886 \\
\hline 44 & 185.03 & 4.861 \\
\hline 46 & 177.89 & 4.839 \\
\hline 48 & 171.29 & 4.818 \\
\hline 50 & 165.16 & 4.799 \\
\hline 52 & 159.45 & 4.781 \\
\hline 54 & 154.13 & 4.765 \\
\hline 56 & 149.15 & 4.750 \\
\hline 58 & 144.47 & 4.736 \\
\hline 60 & 140.09 & 4.723 \\
\hline 62 & 135.95 & 4.710 \\
\hline 63 & 133.98 & 4.704 \\
\hline 64 & 132.06 & 4.699 \\
\hline 66 & .128 .38 & 4.688 \\
\hline 79 & 108.68 & 4.632 \\
\hline 80 & 107.41 & 4.629 \\
\hline 82 & 104.96 & 4.622 \\
\hline 84 & 102.62 & 4.616 \\
\hline 86 & 100.38 & 4.610 \\
\hline 88 & 98.24 & 4.604 \\
\hline 90 & 96.18 & 4.598 \\
\hline 92 & 94.21 & 4.593 \\
\hline 94 & 92.31 & 4.588 \\
\hline 96 & 90.50 & 4.583 \\
\hline 98 & 88.75 & 4.579 \\
\hline 99 & 87.90 & 4.576 \\
\hline 100 & 87.06 & 4.574 \\
\hline 102 & 85.44 & 4.570 \\
\hline 110 & 79.5 & 4.55 \\
\hline 112 & 78.2 & 4.55 \\
\hline 120 & 73.2 & 4.54 \\
\hline 130 & 67.8 & 4.53 \\
\hline
\end{tabular}

Discussion. Calculated periods and phase velocities are given in Tables 4 to 12 and are shown graphically in Figures 4 and 5 . The data shown in Figures 4 and 5 are from recent analyses of traveling and standing waves. It is apparent from an examination of Figures 4 and 5 that no one model adequately explains all the phase velocity data, although it must be re-
TABLE 11. Gutenberg-Birch Model for First Higher Mode

\begin{tabular}{ccc}
\hline Order, $n$ & $T, \mathrm{sec}$ & $c, \mathrm{~km} / \mathrm{sec}$ \\
\hline 72 & 95.44 & 5.785 \\
74 & 93.44 & 5.751 \\
76 & 91.53 & 5.717 \\
78 & 89.70 & 5.685 \\
80 & 87.94 & 5.655 \\
82 & 86.25 & 5.625 \\
84 & 84.64 & 5.597 \\
86 & 83.08 & 5.570 \\
88 & 81.59 & 5.514 \\
90 & 80.15 & 5.519 \\
\hline
\end{tabular}

membered that the data are primarily for oceanic paths.

For periods greater than about 200 seconds all the calculated phase velocities are within 2 per cent of each other, but the data do favor a Gutenberg or Gutenberg-Birch type of mantle structure. It is also interesting to note that for periods greater than 200 seconds the difference between oceanic and continental models is no larger than the differences between several of the continental models.

All the oceanic and continental group velocity curves considered are within $1 \frac{1}{2}$ per cent of each other in the period range $150<T<400$

TABLE 12. CIT6 Model for First Higher Mode

\begin{tabular}{rrr}
\hline Order, $n$ & $T$, sec & $c, \mathrm{~km} / \mathrm{sec}$ \\
\hline 36 & 158.39 & 6.923 \\
38 & 152.40 & 6.821 \\
40 & 146.91 & 6.727 \\
50 & 125.04 & 6.338 \\
52 & 121.50 & 6.274 \\
54 & 118.18 & 6.214 \\
56 & 115.04 & 6.158 \\
58 & 112.09 & 6.104 \\
60 & 109.29 & 6.053 \\
62 & 106.63 & 6.006 \\
64 & 104.11 & 5.960 \\
66 & 101.72 & 5.917 \\
67 & 100.56 & 5.896 \\
68 & 99.44 & 5.876 \\
72 & 95.18 & 5.800 \\
74 & 93.20 & 5.765 \\
76 & 91.30 & 5.731 \\
80 & 87.73 & 5.667 \\
90 & 79.96 & 5.531 \\
92 & 78.58 & 5.507 \\
100 & 73.51 & 5.418 \\
\hline
\end{tabular}




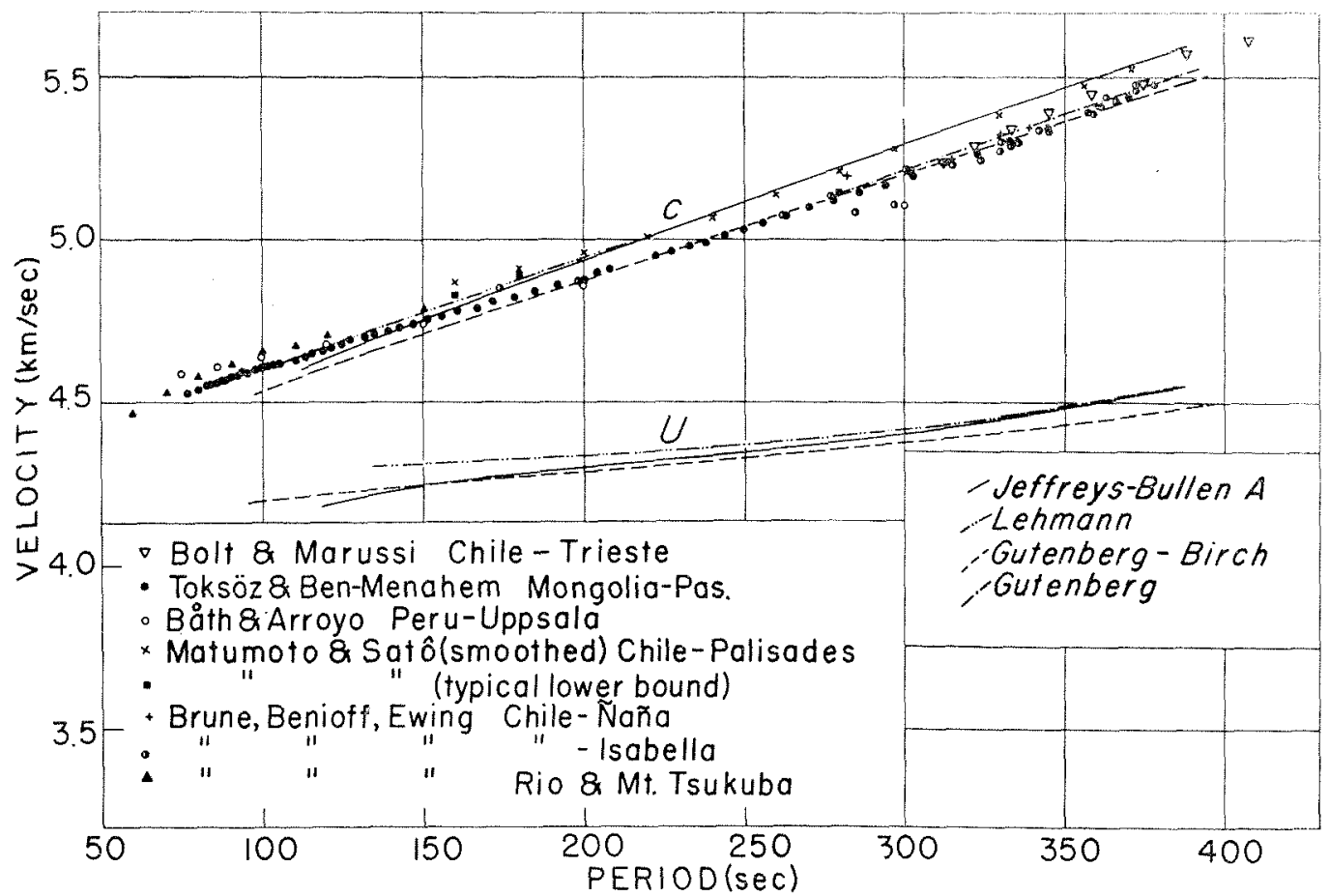

Fig. 4. Love wave dispersion curves for four continental models compared with recent phase velocity data of Toksöz and Ben-Menahem (personal communication) for the Mongolian shock of December 4, 1957, and additional data of Bolt and Marussi [1962]; Baith and Lopez Arroyo [1962] ; Matumoto and Satô [1962]; Brune et al. [1961].

seconds. This fact implies that measurements of group velocity must be made to at least this accuracy in order to differentiate between the various models considered.
Most calculations in the literature have been based on structures of continental type. An oceanic structure is more pertinent if conclusions are to be drawn from free-oscillation or

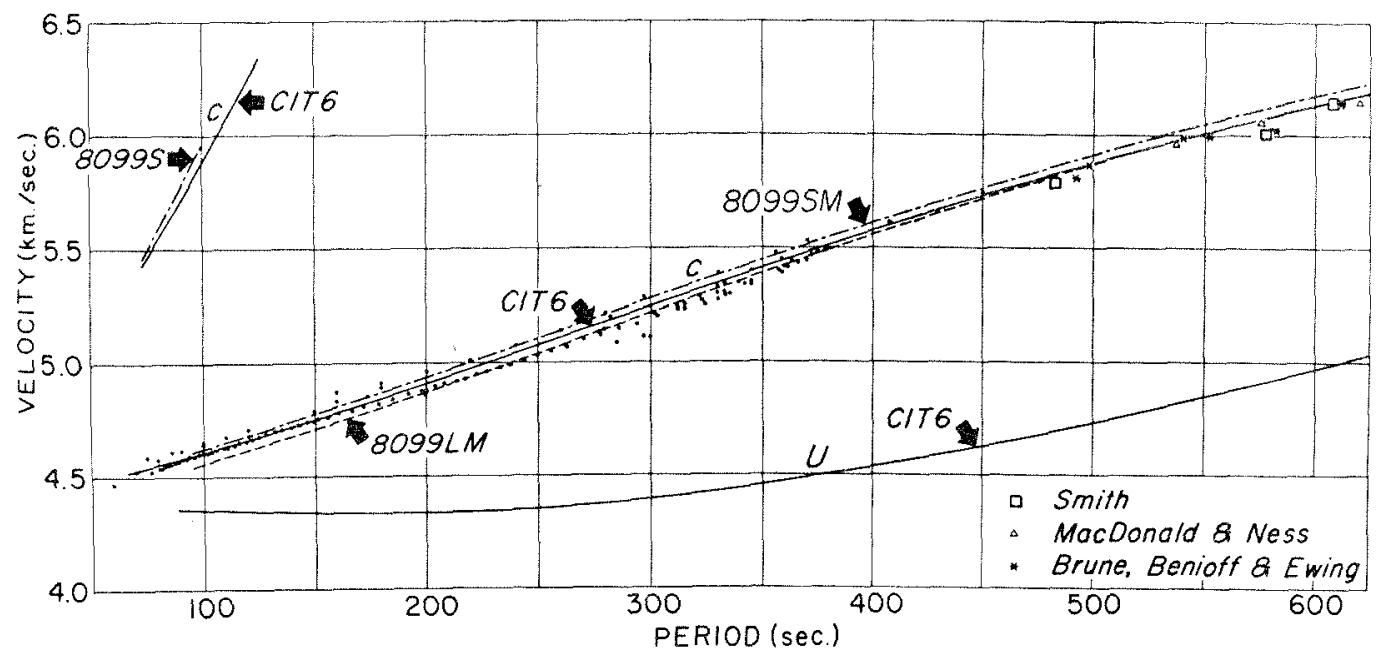

Fig. 5. Love wave dispersion curves for three oceanic models compared with recent phase velocity data. Data are same as in Figure 4 with additional data of Smith [1961], MacDonald and Ness [1961], and Brune et al. [1961]. 


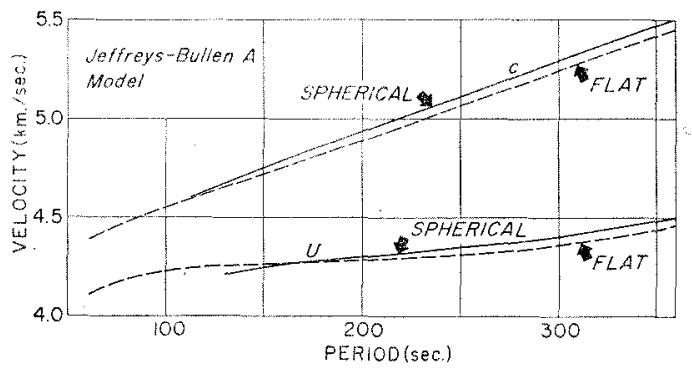

Fig. 6. Effect of sphericity on Love wave dispersion for the Jeffreys-Bullen A model.

world-encircling mantle Love wave data. Dorman et al [1960] developed an oceanic model, designated 8099, which they considered a satisfactory solution on the basis of plane layer calculations. Case 8099 is not a completely satisfactory solution in the light of more recent data for Rayleigh wave phase velocities and spherical earth solutions, but it serves as a convenient reference case. Furthermore, the densities used in 8099 are derived from Jeffrey's velocities and are therefore inconsistent with the actual velocity structure used. The two versions of 8099 considered here are shown in Figure 3 and Table 2. Aside from being two possible oceanic structures, these cases may be considered two extreme methods for approximating the same smooth structure. As is shown in Figure 5, the two structures give quite different dispersion.

CIT6 is a smooth structure with a low-velocity channel and a consistent density. The phase velocity curve for this model falls between 8099SM and 8099LM, although all three eurves fall generally within the scatter of the data. The recent data of Toksöz and BenMenahem (personal communication) favors CIT6 for periods between 60 and about 170 seconds. Between 200 and 350 seconds the data favor 8099LM, and beyond 400 seconds either CIT6 or $8099 \mathrm{LM}$ is satisfactory, although the data scatter. We note that for these long periods the continental Gutenberg structures are equally as satisfactory as the above-mentioned oceanic structures.

Since many previous calculations have been based on plane layered models of the earth, it is important to know how sphericity affects these results. Flat-earth equivalents have been computed for the Jeffreys-Bullen A and CIT6 structures. The resulting dispersion is shown in Figures 6 and 7. The Jeffreys-Bullen A model behaves as expected; the flat and spherical solutions converge at short periods.

For this model we can determine an approximate empirical relation between phase velocities for spherical and plane layered structures.

$$
c \approx c_{h}+0.00016 T
$$

valid to within 0.5 per cent in the period range $100<T<350$ seconds. This correction is good for the Jeffreys-Bullen A model and presumably for similar earth models. Group velocities for this model, computed using flat or spherical

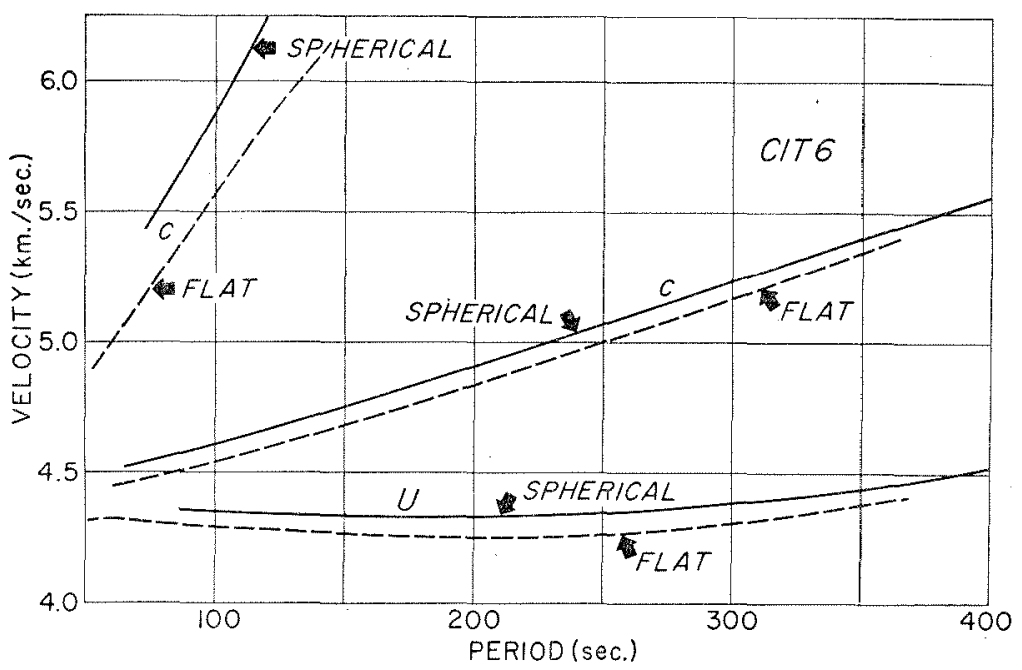

Fig. 7. Effect of sphericity on Love wave dispersion for the CIT 6 model. 


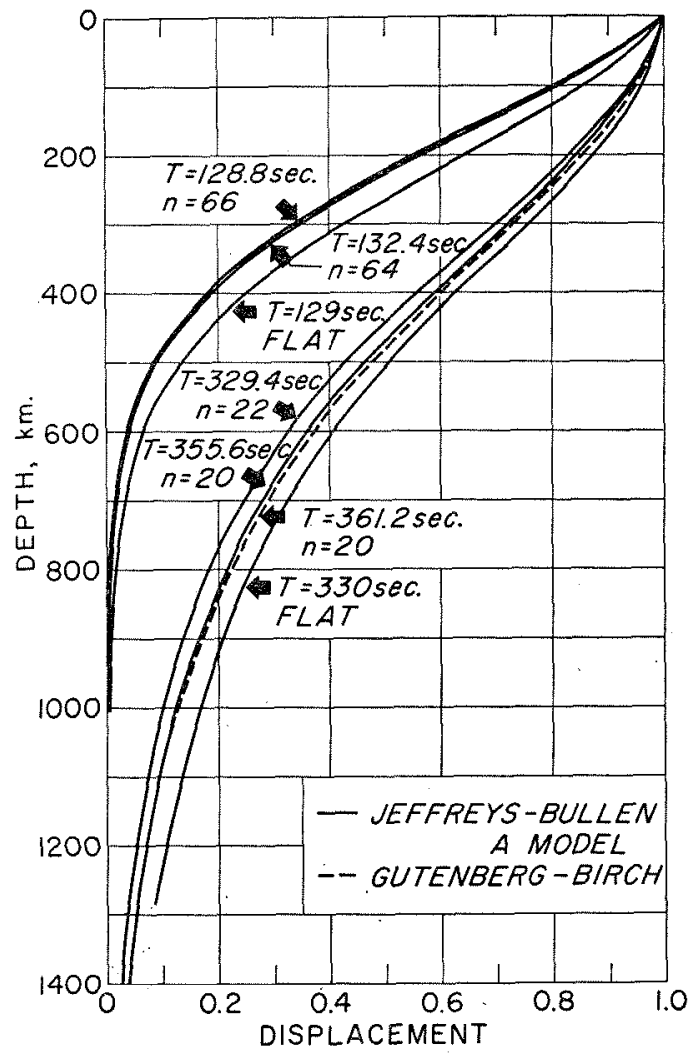

Fig. 8. Comparison of displacements for continental models computed from flat and spherical layer programs.

layers, agree to 1 per cent for periods between 140 and 350 seconds.

A comparison of the calculations for flat and spherical structures for CIT6 gives a somewhat more surprising result. Instead of converging, the two phase velocity curves are almost parallel, the spherical case having phase velocities about $0.065 \mathrm{~km} / \mathrm{sec}$ higher than the equivalent flat case in the period range $70<T<300$ seconds. This can be shown to be due to the presence of the low-velocity channel, which, for $S H$ motion, acts as an efficient energy trap. In a certain range of periods the fundamental mode Love wave is as much a channel mode as a surface mode and is therefore traveling around a smaller sphere.

As can be seen in Figure 5, the effect of sphericity on the first higher Love mode is large. If data for fundamental and higher-mode Love waves are used to determine earth structure, it appears that the effect of sphericity must be included even for periods as short as 20 seconds. However, this situation improves if it can be demonstrated that no low-velocity zone exists in the depth interval of interest.

Displacements. The variations of displacements and stresses with depth are calculated routinely in the process of finding the eigenfrequencies. Displacements and stresses are important not only for checking convergence and verifying mode number but also for determining energies and the resulting effect on dispersion of various sections of the spherical wave guide.

Figure 8 shows the displacements for two ranges of periods for two continental models. The Gutenberg-Birch and the Jeffreys-Bullen A models give quite different dispersion, but the displacements with depth are similar. Displacements for an equivalent flat earth model are greater for the fundamental mode and show that Love waves over a spherical earth do not sample as deep as they do on an equivalent flat earth.

Normalized displacements in the fundamental

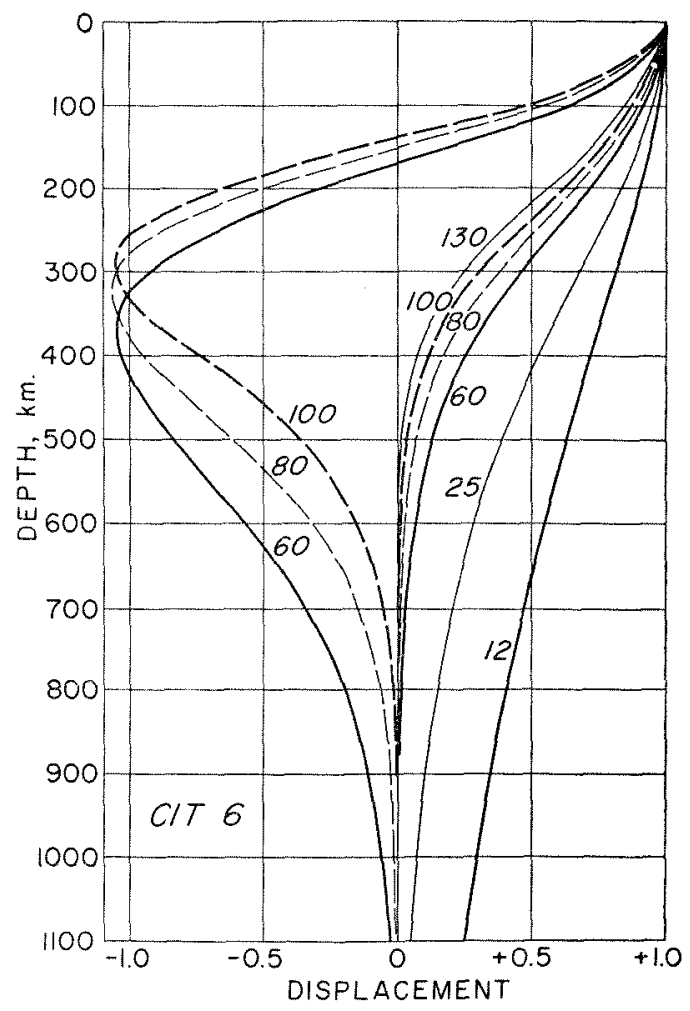

Fig. 9. Displacements for CIT 6 model computed using spherical layer program. 
and first higher Love modes are shown in Figure 9 for the CIT 6 model. The higher modes of a given order number sample successively deeper. Since the higher modes sample the mantle differently than the fundamental mode, the use of higher-mode data promises to be important in determining a unique structure.

Comparisons between displacements in a spherical earth and in a flat earth are made in Figure 10 for both the fundamental and the first higher Love mode. The effect of sphericity is to translate the displacements away from the center of curvature of the displacement-depth function. As is evident from the dispersion (Figure 7) and the variation of displacements with depth, sphericity has a larger effect on highermode Love waves than on the fundamental mode.

Conclusions. In addition to providing theoretical results for mantle Love waves for seven models of a heterogeneous, spherical earth, we are able to draw several important conclusions

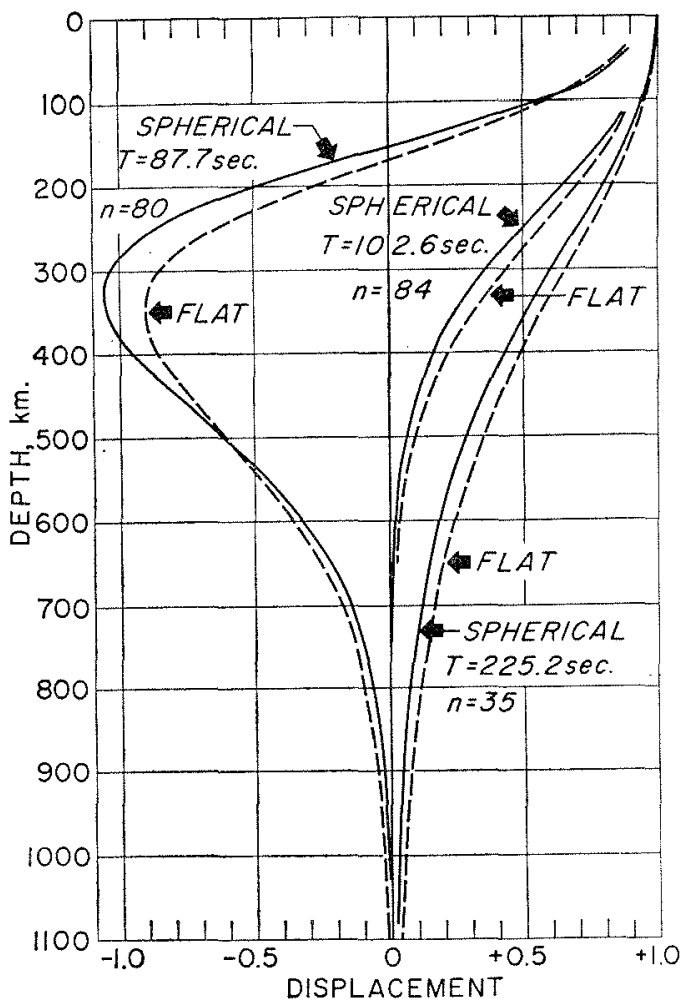

Fig. 10. Comparison of displacements for CIT 6 model computed from flat and spherical layer programs. from our analysis: (1) For the models considered here the effect of sphericity is less extreme, although more complicated, on fundamental mode Love waves of periods greater than some 200 seconds than on Rayleigh waves. A low-velocity channel seems to be more effective in trapping energy for Love waves, and it therefore makes the effect of sphericity show up at very short periods. The sphericity correction is a strong function of earth structure. (2) Calculations of displacement as a function of depth indicate that the first higher Love mode for periods less than 90 seconds is very sensitive to the upper mantle structure in the vicinity of the low-velocity zone and is a potentially useful source of information for analyzing the details of this region. (3) The data seem to favor a CIT6 oceanic upper mantle structure and a Gutenberg or Gutenberg-Birch lower mantle structure. It is preferable to use a Gutenberg-Birch structure, however, because of the consistent velocity-density relation. (4) For periods greater than 200 seconds the difference between the dispersion for oceanic and continental structures is no greater than the difference between the dispersion for various proposed continental models. (5) The group velocity of mantle Love waves is much less sensitive to different structures than the phase velocity is. (6) More precise and consistent experimental data for Love wave dispersion are needed before the question of the best model for the earth's mantle can be resolved.

Acknowledgments. We are grateful to Dr. Russell $\mathrm{E}$. Carr for discussing various aspects of the theoretical solution. Mr. M. Nafi Toksöz and Dr. Ari Ben-Menahem kindly allowed us to use their data in advance of publication.

This paper presents research performed at the Jet Propulsion Laboratory and the Seismological Laboratory, California Institute of Technology, under contracts NASw-6 and NASw-81, sponsored by the National Aeronautics and Space Administration, and contract AF-49(638)910 of the Air Force Office of Scientific Research as part of the Advanced Research Projects Agency, project Vela.

\section{REFERENCES}

Aki, K., and F. Press, Upper mantle structure under oceans and continents from Rayleigh waves, Geophys. J., 5, 292-305, 1961.

Alterman, Z., H. Jarosch, and C. L. Pekeris, Oscillations of the earth, Proc. Roy. Soc., London, A, 252, 80-95, 1959.

Alterman, Z, H. Jarosch, and C. L. Pekeris, Propa- 
gation of Rayleigh waves in the earth, Geophys. J., 4, 219-241, 1961.

Anderson, D. L., and D. Harkrider, The effect of anisotropy on continental and oceanic surface wave dispersion (abstract), J. Geophys. Research, $67,1627,1962$.

Batth, M., and A. Lopez Arroyo, Attenuation and dispersion of $G$ waves, J. Geophys. Research, 67 , 1933-1942, 1962.

Birch, F., Composition of the earth's mantle, Geophys. J., 4, 295-311, 1961.

Bolt, B., and J. Dorman, Phase and group velocities of Rayleigh waves in a spherical, gravitating earth, J. Geophys. Research, 66, 2965-2981, 1961.

Bolt, B., and A. Marussi, Eigenvibrations of the earth observed at Trieste, Geophys. J., 6, 299$311,1962$.

Brune, J., H. Benioff, and M. Ewing, Long-period surface waves from the Chilean earthquake of May 22, 1960, recorded on linear strain seismographs, J. Geophys. Research, 66, 2895-2910, 1961 .

Bullard, E. C., The density within the earth, Verhandel. Ned. Geol. Mijnbouwk. Genoot., Geol. Ser., 18, 23-41, 1957.

Carr, R., Free oscillations of a gravitating solid sphere, Tech. Rept. 92-164, Jet Propulsion Laboratory, California Institute of Technology, Pasadena, 1961.

Carr, R., and R. L. Kovach, Toroidal oscillations of the moon, lcarus, 1, 75-76, 1962.

Dorman, J., M. Ewing, and J. Oliver, Study of shear velocity distribution by mantle Rayleigh waves, Bull. Seismol. Soc. Am., 50, 87-115, 1960.

Ewing, M., and F. Press, An investigation of manthe Rayleigh waves, Bull. Seismol. Soc. Am., 44, $127-148,1954 \pi$.

Ewing, M., and F. Press, Mantle Rayleigh waves from the Kamchatka earthquake of November 4, 1952, Bull. Seismol. Soc. Am., 44, 471-479, $1954 b$.

Gilbert, F., and G. J. F. MacDonald, Free oscillations of the earth, 1 , Toroidal oscillations, $J$. Geophys. Research, 65, 675-693, 1960.

Gutenberg, B., The asthenosphere low velocity layer, Ann. geofis., Rome, 12, 439-460, 1959.

Hoskins, L. M., The strain of a gravitating sphere of variable density and elasticity, Trans. Am. Math. Soc., 21, 1-43, 1920.

Jeans, J. H., The propagation of earthquake waves,
Proc. Roy. Soc., London, A, 102, 554-574, 1923.

Jobert, N., Évaluation de la période d'oscillation d'une sphère élastique heterogene, par applicam tion du principe de Rayleigh, Compt. rend., 243, $1230-1232,1956$

Jobert, N., Calcul de la dispersion des ondes de Love de grande période à la surface de la terre, Ann. géophys., 16, 393-413, 1960.

Kobayashi, N., and H. Takeuchi, Surface waves propagating along the free surface of a semiinfinite elastic medium of variable density and elasticity, 5, Mantle Love waves, Geophys. Notes, Tolyo Univ., 14, 232-240, 1961.

Lehmann, $\mathrm{I}$., The times of $P$ and $S$ in northeastern America, Ann. geofus., Rome, 8, 351-370, 1955.

Love, A. E. H., Some Problems of Geodynamics, Cambridge University Press, 1911.

MacDonald, G. J. F., and N. F. Ness, A study of the free oscillations of the earth, $J$. Geophys. Research, 66, 1865-1912, 1961.

Matumoto, T., and Y. Satô, Phase velocity of longperiod Rayleigh and Love waves as observed in the Chilean earthquake of May 22, 1960 (abstract), J. Geophys. Research, 67 (9), 3579, 1962.

Pekeris, C. L., Z. Alterman, and H. Jarosch, Comparison of theoretical with observed values of the periods of the free oscillations of the earth, Proc. Natl. Acad. Sci. U. S., 47, 91-98, 1961.

Satô, Y., M. Landisman, and M. Ewing, Love waves in a heterogeneous, spherical earth, J. Geophys. Research, 65, 2395-2404, 1960.

Smith, S., An investigation of the earth's free oscillations, Ph.D. thesis, California Institute of Technology, Pasadena, 1961.

Stoneley, R., The oscillations of the earth, in Physics and Chemistry of the Earth, vol. 4, Pergamon Press, London, 1961.

Takeuchi, H., Torsional oscillations of the earth and some related problems, Geophys. J., 2, 89$100,1959$.

Takeuchi, H., F. Press, and N. Kobayashi, Rayleigh wave evidence for the low velocity zone in the mantle, Bull. Seismol. Soc. Am., 49, 355364,1959

Takeuchi, H., M. Saito, and N. Kobayashi, Free oscillations of the moon, J. Geophys. Research, $66,3895-3897,1961$.

(Manuscript received July 27, 1962; revised September 5, 1962.) 